\title{
Penehyclidine hydrochloride inhibits TLR4 signaling and inflammation, and attenuates blunt chest trauma and hemorrhagic shock-induced acute lung injury in rats
}

\author{
XIAO-JING WU ${ }^{1 *}$, HUI-MIN LIU ${ }^{1 *}$, XUE-MIN SONG ${ }^{2}$, BO ZHAO ${ }^{1}$, YAN LENG $^{1}$, E-YOU WANG ${ }^{1}$, \\ LI-YING ZHAN $^{1}$, QING-TAO MENG ${ }^{1}$ and ZHONG-YUAN XIA ${ }^{1}$ \\ ${ }^{1}$ Department of Anesthesiology, Renmin Hospital of Wuhan University, Wuhan, Hubei 430060; \\ ${ }^{2}$ Department of Anesthesiology and Critical Care Medicine, Zhongnan Hospital of Wuhan University, \\ Wuhan, Hubei 430071, P.R. China
}

Received June 3, 2017; Accepted January 11, 2018

DOI: $10.3892 / \mathrm{mmr} .2018 .8644$

\begin{abstract}
Blunt chest trauma with hemorrhagic shock (THS) frequently induces pulmonary inflammation that leads to acute lung injury (ALI). Penehyclidine hydrochloride (PHC) possesses anti-inflammatory properties that may attenuate the systemic inflammatory response. The present study aimed to evaluate the molecular mechanism of PHC in modifying THS-induced ALI in rats. Rats underwent either THS or a sham procedure. At $6 \mathrm{~h}$ subsequent to blunt chest trauma, arterial blood was drawn for blood gas and pro-inflammatory factors analyses, and lung tissue samples were collected to examine pulmonary histopathological alterations, the wet/dry weight ratio, myeloperoxidase activity, and the protein expression levels of Toll-like receptor 4 (TLR4), phosphorylated (p-)p38 mitogen-activated protein kinase (MAPK), nuclear factor (NF)- $\mathrm{kB}$ and activator protein-1 (AP-1). THS caused significant reductions in heart rate and mean arterial blood pressure, and was associated with significant increases in tumor necrosis factor- $\alpha$, interleukin (IL)-6, IL-1 $\beta$, p-p38MAPK, NF- $\kappa \mathrm{B}$ and AP-1 activation, in addition to TLR4 expression, in the lung. PHC effectively attenuated THS-induced
\end{abstract}

Correspondence to: Professor Zhong-Yuan Xia, Department of Anesthesiology, Renmin Hospital of Wuhan University, 238 Liberation Road, Wuhan, Hubei 430060, P.R. China

E-mail: xiazhongyuanmz@hotmail.com

*Contributed equally

Abbreviations: PHC, penehyclidine hydrochloride; ALI, acute lung injury; THS, blunt chest trauma with hemorrhagic shock; HS, hemorrhagic shock; AP-1, activator protein-1; TNF, tumor necrosis factor; NF- $\kappa \mathrm{B}$, nuclear factor- $\kappa \mathrm{B}$; IL, interleukin; MAPKs, mitogen-activated protein kinases; TLRs, Toll-like receptors; ARDS, acute respiratory distress syndrome; MPO, myeloperoxidase; IQA, index of quantitative assessment

Key words: PHC, THS, ALI, TLR4, p38MAPK, NF-кB, AP-1
ALI, and inhibited TLR4 expression, reduced the activation of p-p38MAPK, NF- $\mathrm{B}$ and AP-1, and downregulated the expression of pro-inflammatory mediators. In conclusion, the results of the present study demonstrated that PHC may exert an anti-inflammatory effect and attenuate THS-induced ALI by inhibiting the TLR4 signaling pathway. These preclinical findings may offer a novel therapeutic strategy to restrict TLR4 overactivation in ALI.

\section{Introduction}

Blunt chest trauma frequently occurs in patients with poly-trauma, including victims of vehicular accidents, and is frequently associated with hemorrhagic shock (HS) (1). Blunt chest trauma with HS (THS) is associated with a high risk of acute lung injury (ALI) and acute respiratory distress syndrome (ARDS), which results in considerable morbidity and mortality (2). Previous studies have demonstrated that ALI due to THS is associated with a progressive inflammatory response during the development of the ALI $(3,4)$. Toll-like receptors (TLRs) are innate immune receptors that serve a crucial role in the regulation of inflammatory and innate immune responses. Among these receptors, Toll-like receptor 4 (TLR4) is specifically recognized to be a candidate gene that is involved with distinct forms of ALI (5). TLR4 may activate multiple intracellular signaling systems, including the p38 mitogen-activated protein kinase (p38MAPK) and nuclear factor- $\kappa \mathrm{B}(\mathrm{NF}-\kappa \mathrm{B})$ pathways, and induce the expression of pro-inflammatory cytokines, including tumor necrosis factor- $\alpha$ (TNF- $\alpha)$, interleukin- 6 (IL-6), IL-1 $\beta$ and additional pro-inflammatory mediators involved in ALI. MAPKs are well known as important mediators of inflammation-induced tissue injury. Notably, MAPK activation has been studied in inflammation-associated lung dysfunction and has been demonstrated to contribute to ALI. In addition, p38MAPK is known to be important for the expression of components of activator protein-1 (AP-1) $(6,7)$. However, the potential involvement of TLR4-mediated MAPK phosphorylation following THS-induced ALI has not been examined. 
Penehyclidine hydrochloride (PHC), a novel anticholinergic drug which is derived from hyoscyamine, has been reported to attenuate systemic inflammatory responses and exhibit anti-apoptotic properties (8). Increasing basic and clinical evidence has indicated that PHC may suppress pro-inflammatory cytokine production. Zhan et al (9) reported that PHC preconditioning may exert protective effects by inhibiting inflammatory factor production and suppressing p38MAPK activation in septic mice. Additionally, Li et al (10) reported that treatment with $\mathrm{PHC}$ reduced the level of pro-inflammatory cytokines and suppressed lipid peroxidation in rats with ARDS. Li et al (11) discovered that PHC exhibited marked effects on inhibiting the upregulation of inflammatory molecules downstream of the TLR4 signaling pathway in patients with traumatic ALI. Although a number of studies have addressed the therapeutic potential of PHC, whether PHC can protect against ALI from THS remains unknown. A 2012 study (12) reported the protective effect of PHC on pulmonary contusion induced by blunt chest trauma in rats; in that study, a rat model of blunt chest trauma alone was used.

In the present study, a novel combination rat model of THS was used. The present study elucidated the effects of PHC on THS-induced ALI, which inhibited TLR4 signaling and inflammation in the rats. The important proteins and transcription factors of the TLR4 signaling pathway (p-p38MAPK, $\mathrm{NF}-\kappa \mathrm{B}$ and AP-1) were additionally examined.

\section{Materials and methods}

Animals andreagents. A totalof 30 healthy male Sprague-Dawley rats (aged 8 weeks, weighing 240-280 g) were obtained from Hunan Institute for Biological Sciences (Hunan, China; certificate no. SCXX 2009-0004) and maintained under specific pathogen-free conditions. Rats were housed in conditions of a 12/12 h light/dark cycle, a mean room temperature of $22-24^{\circ} \mathrm{C}$, and a humidity of $50-60 \%$. All animal experiments complied with the Guide for the Care and Use of Laboratory Animals of the National Institutes of Health (Bethesda, MD, USA), and were approved by the Bioethics Committee of Renmin Hospital of Wuhan University (Wuhan, China). PHC was purchased from Chengdu List Pharmaceutical Co., Ltd. (Chengdu, China). TLR4 and phosphorylated (p)-p38MAPK antibodies were purchased from Cell Signaling Technology, Inc. (Danvers, MA, USA). ELISA kits were obtained from BD Biosciences (BD Pharmingen; BD Biosciences, San Jose, CA, USA) and myeloperoxidase (MPO) kits were purchased from Nanjing Jiancheng Bioengineering Institute (Nanjing, China).

THS model. The present study used a novel combination rat model of THS previously described by Raghavendran et al (13) and Knöferl et al (14). Rats were anesthetized with intraperitoneal sodium pentobarbital $(50 \mathrm{mg} / \mathrm{kg})$ injections and subjected to sham or THS surgery. The femoral artery and vein were cannulated with polyethylene tubing for continuous invasive pressure monitoring and to establish venous access. Heart rate (HR) and mean arterial blood pressure (MAP) were determined using Philips IntelliVue MP40 (Philips Healthcare, Andover, MA, USA). Blunt chest trauma was induced in anesthetized rats at a fixed chest impact energy of $2.45 \mathrm{~J}$, as described previously (12). Following 1-3 min, HS was induced by blood withdrawal through the tubing, which was not attached to the monitor until the MAP reached $35-40 \mathrm{mmHg}$. This pressure was maintained for $1 \mathrm{~h}$. During the next $1 \mathrm{~h}$, the rats were resuscitated by transfusion of the withdrawn blood, and the withdrawn blood volume was infused twice in the form of Ringer's lactate solution (Baxter Healthcare Co., Ltd., Tianjin, China).

Experimental protocols. A total of 30 rats were randomly assigned to three equal groups ( $\mathrm{n}=10$ rats/group): The sham group, THS group and PHC group. In the PHC group, the rats were infused with PHC at $2 \mathrm{mg} / \mathrm{kg}$ for $30 \mathrm{~min}$ prior to the induction of blunt chest trauma. The sham control and THS rats received the same volume of $0.9 \%$ normal saline solution. The sham control animals were subjected to the same experimental procedures, including cannulation of the femoral artery and vein, although no blunt chest trauma or HS was induced. All animals were sacrificed under anesthesia via intraperitoneal injection of sodium pentobarbital $(50 \mathrm{mg} / \mathrm{kg})$ and exsanguination from the right carotid artery at $6 \mathrm{~h}$ post-THS challenge. Blood samples and lung tissue specimens were harvested.

Blood gas analysis and lactic acid. Arterial blood was assayed following collection from the right carotid artery $(1.0 \mathrm{ml}$ each) when the animal was sacrificed by exsanguination. Arterial blood samples were analyzed for $\mathrm{pH}$, partial pressure of oxygen $\left(\mathrm{PaO}_{2}\right), \mathrm{PaCO}_{2}, \mathrm{PaO}_{2} /$ fraction of inspired oxygen $\left(\mathrm{FiO}_{2}\right)$ and lactic acid, which were immediately determined using an i-STAT Portable Clinical Analyzer (Abbott Point of Care Inc., Princeton, NJ, USA).

Measurement of the lung wet/dry weight (W/D) ratio and $M P O$ activity. The water content of the lungs was determined by calculating the W/D ratio of lung tissues at $6 \mathrm{~h}$ post-THS challenge. The right lower lobe of the lung was dissected free from nonpulmonary tissues, and weighed and dried in an oven at $60^{\circ} \mathrm{C}$ for $72 \mathrm{~h}$, followed by reweighing. The W/D ratios are reported as a measure of pulmonary edema.

MPO activity was determined as an index of neutrophil accumulation in the lungs. Frozen $\left(4^{\circ} \mathrm{C}\right)$ lung tissues were homogenized and centrifuged at $1,500 \mathrm{x} \mathrm{g}$ for $10 \mathrm{~min}$ at $4^{\circ} \mathrm{C}$. Following weighing, the lungs were homogenized, centrifuged $\left(1,000 \mathrm{xg}\right.$ for $30 \mathrm{~min}$ at $\left.4^{\circ} \mathrm{C}\right)$ and resuspended in $50 \mathrm{mM} \mathrm{KH}_{2} \mathrm{PO}_{4}$ buffer (Ph 6.0; cat. no. p0662; Sigma-Aldrich; Merck KGaA, Darmstadt, Germany) with $0.5 \%$ hexadecyltrimethylammonium bromide (cat. no. H5882; Sigma-Aldrich; Merck KGaA). Subsequently, samples were sonicated for $1 \mathrm{~min}$ at $4^{\circ} \mathrm{C}$ and $20 \mathrm{KHz}$, and then incubated at $60^{\circ} \mathrm{C}$ for $2 \mathrm{~h}$. The absorbance of visible light at $460 \mathrm{~nm}$ was measured and the MPO activity was calculated in units/gram of lung tissue. The MPO content was determined by following the manufacturer's protocol for the MPO assay kit. The results are expressed as units/gram of protein/minute (U/g).

Transmission electron microscopy (TEM). The fragments of the right middle-lung tissue were cut into 1-mm-thick slices, immersion-fixed in $2.5 \%$ buffered glutaraldehyde at $0-4^{\circ} \mathrm{C}$ for $2 \mathrm{~h}$, buffered in PBS three times, fixed with $1 \%$ osmic acid for $1 \mathrm{~h}$ at room temperature, washed with distilled water and dehydrated with dimethylketone. Following embedding in 
Epon-812, they were cut into ultrathin sections $(60 \mathrm{~nm})$ using an LKB-V ultramicrotome (LKB Produkter AB; Bromma, Stockholm, Sweden) and stained with $1 \%$ uranyl acetate for $30 \mathrm{~min}$ at room temperature and lead citrate for $15 \mathrm{~min}$ at room temperature. The sections were examined using a Hitachi H-600 transmission electron microscope (Hitachi, Ltd., Tokyo, Japan).

Hematoxylin and eosin $(H \& E)$ staining. All animals were sacrificed via exsanguination from the right carotid artery at $6 \mathrm{~h}$ post-THS, and lung tissue samples were harvested immediately. The right middle-lung specimens were fixed in $10 \%$ formalin at $4^{\circ} \mathrm{C}$ for $24 \mathrm{~h}$, sectioned $(5 \mu \mathrm{m})$ and then stained with $\mathrm{H} \& \mathrm{E}$ at room temperature. Following this, paraffin sections were incubated at $60^{\circ} \mathrm{C}$ for $30 \mathrm{~min}$, twice immersed in xylene for $15 \mathrm{~min}$ at room temperature and then treated with a descending ethanol series (100, 95, 90, 85 and 75\%) for 5 min each at room temperature. The sections were then treated with $0.5 \%$ hematoxylin for $1-5 \mathrm{~min}$ at room temperature and then rinsed in tap water for $1 \mathrm{~min}$. Sections were incubated with PBS for $8 \mathrm{sec}$ until a blue color was observed, and then the sections were washed using tap water for $1 \mathrm{~min}$ and then distilled water for $8 \mathrm{sec}$. Sections were then stained with $1 \%$ eosin for $3 \mathrm{~min}$ at room temperature and then washed with tap water. Following this, sections were treated with an ascending ethanol series $(75,85,90$ and $95 \%)$ for $1 \mathrm{~min}$ each at room temperature. Sections were then analyzed and graded using the index of quantitative assessment (IQA) for the presence of interstitial neutrophilic infiltrates, intra-alveolar hemorrhage and pulmonary edema using a TEM microscope (magnification, x200; BX51; Olympus Corporation, Tokyo, Japan) according to the aforementioned protocol. Upon viewing $\sim 10$ fields/sector under low and high power, each section was assigned a numerical histological IQA of lung injury using the following criteria: 0 , normal; +1 , focal epithelial edema, pleural-based lesions occupying $<25 \%$ of the lung; +2 , diffuse swelling with villi necrosis, more extensive fibrosis involving $26-50 \%$ of the lung and fibrotic regions; +3 , diffuse pathology, neutrophil infiltration and widespread fibrosis involving $51-70 \%$ of the lung; and +4 , major widespread injury with massive neutrophil infiltration and hemorrhage, and widespread fibrosis involving $>70 \%$ of the lung.

Western blot analysis. Western blot analysis was used to determine TLR4 (rabbit anti-mouse) and p-p38MAPK kinase (rabbit anti-mouse) activity in the lungs. Lung tissue samples were thawed and suspended in homogenization buffer $(25 \mathrm{mM}$ Tris-HCl, pH 7.6, 1\% NP-40, 0.5\% sodium deoxycholate and $0.1 \%$ SDS) and homogenized. The homogenate was centrifuged at $3,000 \mathrm{x} \mathrm{g}$ at $4^{\circ} \mathrm{C}$ for $10 \mathrm{~min}$, and the supernatant was centrifuged again at $10,000 \mathrm{x} \mathrm{g}$ at $4^{\circ} \mathrm{C}$ for $10 \mathrm{~min}$. Solubilized protein concentrations were determined using a bicinchoninic acid (BCA) protein assay kit and equal amounts of protein $(10 \mu \mathrm{m} / \mathrm{mg})$ were loaded per well on a $10 \%$ SDS-PAGE gel. Subsequently, the proteins were transferred onto a polyvinylidene difluoride membrane. Blots were blocked overnight at $4^{\circ} \mathrm{C}$ with TBS containing $0.1 \%$ Tween-20 and 5\% non-fat milk, and subsequently incubated with rabbit anti-TLR4 (1:500; cat. no. ab13556; Abcam, Shanghai, China) and p-p38 MAPK rabbit mAb (1:1,000; cat. no. 9215s; Cell Signaling
Technology, Inc.) separately at room temperature for $1 \mathrm{~h}$ followed by incubation with secondary antibody goat anti-rabbit IgG-HRP (1:2,000; cat. no. sc-2004; Santa Cruz Biotechnology, Inc., Dallas, TX, USA) at room temperature for $1 \mathrm{~h}$. The blots were stripped with stripping buffer [2-metaptoethanol $(35 \mu \mathrm{l}), 10 \% \operatorname{SDS}(1 \mathrm{ml})$, Tris $(0.5 \mathrm{M}, \mathrm{pH} 6.7,625 \mu \mathrm{l})$ and $\left.\mathrm{dH}_{2} \mathrm{O}(3.34 \mathrm{ml})\right]$ and reprobed with rabbit anti-rat GAPDH (1:2,000; cat. no. 51332; Cell Signaling Technology, Inc.) at room temperature for $1 \mathrm{~h}$. The immunore active proteins were detected and densitometric analysis was performed using the Odyssey ${ }^{\circledR}$ Fc Imaging System (LI-COR Biosciences, Lincoln, NE, USA).

Immunofluorescence staining and quantitative analysis. The lungs of rats were flushed with ice-cold PBS, and then fixed with $10 \%$ formalin overnight at $4^{\circ} \mathrm{C}$. Paraffin-embedded lung sections $(5 \mu \mathrm{m})$ were stained using the streptavidin-biotin complex immunofluorescence technique for $\mathrm{p}$-p38MAPK detection as follows: The sections were then dewaxed in xylene three times for $5 \mathrm{~min}$ each at room temperature and rehydrated in a descending series of ethanol (100 and 95\%) at room temperature for $10 \mathrm{~min}$ each. Following deparaffinization, sections were brought to a boil in $10 \mathrm{mM}$ sodium citrate buffer ( $\mathrm{pH}$ 6.0) and then maintained at a sub-boiling temperature for $10 \mathrm{~min}$. Slides were then cooled for $30 \mathrm{~min}$ at room temperature. Sections were then blocked using blocking buffer [1X PBS/5\% normal goat serum (cat. no. 5425; Cell Signaling Technology, Inc.)/0.3\% Triton X-100] for $1 \mathrm{~h}$ at room temperature to minimize non-specific staining and then incubated overnight at $4^{\circ} \mathrm{C}$ with p-p38MAPK rabbit mAb (1:50; cat. no. 8632; Cell Signaling Technology, Inc.). Following washing with PBS three times at room temperature for 5 min each, sections were incubated with anti-rabbit IgG (1:1,000; cat. no. 8889; Cell Signaling Technology, Inc.) for $30 \mathrm{~min}$ at room temperature in the dark. Red staining in the nucleus and cytoplasm was considered to be an indicator of positive expression. The mean optical densities of p-p38 MAPK-positive cells from each section were analyzed by image cytometry using HIPAS-2000 image analysis software (Wuhan Qianli Technical Imaging Co. Ltd., Wuhan, China). Using the HIPAS-2000 software, the results were evaluated semi-quantitatively according to the optical density values of positive expression.

Nuclear protein extraction and electrophoretic mobility shift assays. Nuclear extracts were prepared from lung tissues using Nuclear Extraction Reagent, according to the manufacturer's protocol (Viagene Biotech, Inc., Tampa, FL, USA), and aliquots were incubated with $\gamma^{-32} \mathrm{P}$-ATP-labelled oligonucleotides containing the binding sites for NF- $\kappa \mathrm{B}\left(5^{\prime}\right.$-AGTTGA GGGGACTTTCCCAGGC-3') and AP-1 (5'-CGCTTGATG AGTCAGCCGGAA-3') (Pierce; Thermo Fisher Scientific, Inc.). The protein content was measured via the BCA assay. DNA-protein complexes were separated on a $4 \%$ nondenaturing polyacrylamide gel at $90 \mathrm{~V}$ for $1.5 \mathrm{~h}$. The gels were dried, autoradiographed, and quantified using phosphor-imager analysis (Santa Cruz Biotechnology, Inc.).

Determination of TNF- $\alpha, I L-6$ and $I L-1 \beta$ in the serum. Following collection of arterial blood samples, the blood 
was immediately separated by centrifugation at $1,500 \mathrm{x} \mathrm{g}$ for $15 \mathrm{~min}$ at $4^{\circ} \mathrm{C}$. The serum was divided into aliquots and stored at $-80^{\circ} \mathrm{C}$ until the assay. Cytokines (TNF- $\alpha$, IL- 6 and IL-1 $\beta$ ) were measured using commercially available ELISA kits (cat. nos. 558532, 550319 and 559603, respectively; BD Biosciences), according to the manufacturer's protocol. The absorbance of each well was read at $450 \mathrm{~nm}$ using an ELISA plate reader.

Statistical analysis. The data are presented as the mean \pm standard deviation. Data analysis was performed using SPSS 17.0 software (SPSS, Inc., Chicago, IL, USA). Differences associated with the primary sources of variation were tested with one-way analysis of variance (ANOVA). When the F-statistic was significant in the ANOVA comparisons, differences between individual means were tested for significance using the Bonferroni test. The Bonferroni test is a post hoc test that adjusts the $\alpha$ for multiple comparisons. Each experiment was replicated thrice. $\mathrm{P}<0.05$ was considered to indicate a statistically significant difference.

\section{Results}

Hemodynamics (HR and MAP). HR and MAP in the three groups of rats were observed at the different time points. No significant alterations in MAP and HR were observed in the sham control group at $6 \mathrm{~h}$. At 2 and $4 \mathrm{~h}, \mathrm{MAP}$ and HR were decreased significantly in the THS rats $(\mathrm{P}<0.05)$ compared with those in the sham controls, whereas fluctuations in MAP and HR were markedly attenuated following the infusion of $2 \mathrm{mg} / \mathrm{kg}$ of PHC (Fig. 1).

Effects of PHC on blood gas and lactic acid in THS-induced ALI rats. $\mathrm{PaO}_{2} / \mathrm{FiO}_{2}$, as an evaluation index of gas exchange, was measured to determine the degree of lung injury at $6 \mathrm{~h}$ post-THS challenge. The rats in the THS group exhibited a significant decrease in arterial blood $\mathrm{PaO}_{2}$, and notable increases in arterial blood $\mathrm{PaCO}_{2}$ and lactic acid $(\mathrm{P}<0.05)$. Furthermore, the $\mathrm{pH}$ and the $\mathrm{PaO}_{2} / \mathrm{FiO}_{2}$ ratio in the arterial blood were decreased $(\mathrm{P}<0.05)$. Pretreatment with $\mathrm{PHC}$ efficiently reversed the decreases in $\mathrm{PaO}_{2}, \mathrm{pH}$ and $\mathrm{PaO}_{2} / \mathrm{FiO}_{2}$, and attenuated the increases in $\mathrm{PaCO}_{2}$ and $\mathrm{Lac}$ induced by the THS challenge (Fig. 2).

Effects of PHC on the lung W/D ratio and MPO activity in THS-induced ALI rats. Neutrophil accumulation in the lung tissue and organ edema were evaluated by MPO assay and the W/D ratio, respectively. As presented in Fig. 3, the lung W/D ratio and MPO activity were significantly elevated at $6 \mathrm{~h}$ following ALI due to the THS challenge, compared with those of the sham control animals $(\mathrm{P}<0.05)$. Treatment with PHC caused a significant decrease in the W/D ratio and MPO activity ( $\mathrm{P}<0.05$ vs. THS) (Fig. 3).

Effects of PHC on THS-mediated lung pathological alterations. As presented in Fig. 4A, the hematoxylin and eosin staining microscopic findings in the lung sections revealed a normal lung parenchyma in the sham group. In the THS group, the normal alveolar structure of the rat lung was disrupted, with severe hemorrhage and congestion with infiltrating leukocytes.
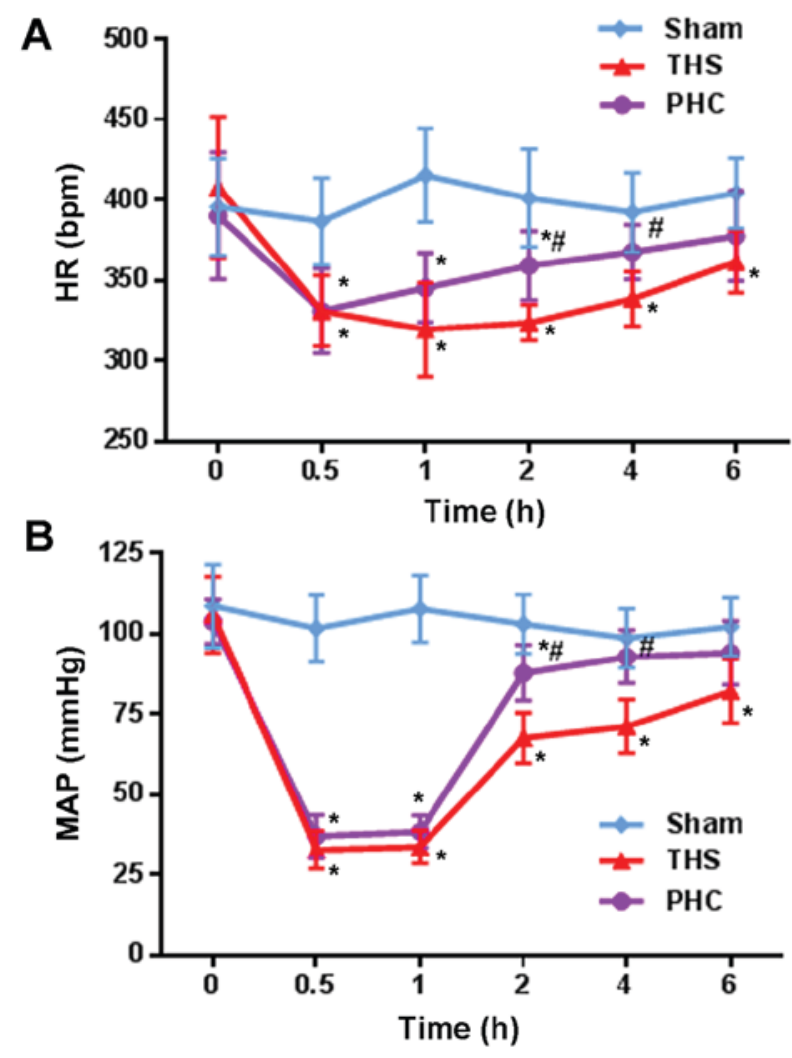

Figure 1. Hemodynamics (HR and MAP). Alterations in (A) HR and (B) MAP in the three groups of rats over the observation period. ${ }^{*} \mathrm{P}<0.05$ vs. sham group; ${ }^{\#} \mathrm{P}<0.05$ vs. THS group. The data are presented as the mean \pm standard deviation $(n=10)$. HR, heart rate; MAP, mean arterial pressure; THS, blunt chest trauma with hemorrhagic shock; PHC, penehyclidine hydrochloride.

By contrast, the rats in the PHC group exhibited significantly less hemorrhaging and leukocyte infiltration compared with the THS group. The IQA scores were 0.5, 3.2 and 2.3 in the sham, THS and PHC groups, respectively (Fig. 4B). The IQA scores in the THS group were increased compared with those in the sham control group $(\mathrm{P}<0.05)$, and the PHC group $(\mathrm{P}<0.05)$.

In Fig. 5, electron microscopy of the THS rat tissues demonstrated ridge dissolution and mitochondrial vacuolization in certain vascular endothelial cells and alveolar epithelial cells, in addition to emptied lamellar bodies. Furthermore, in the THS group, mitochondria in type II alveolar cells appeared swollen, osmiophilic lamellar bodies were emptied, and the cellular ridge had lodged and disappeared. Additionally, the conjunctions between the alveolar epithelial cells and the capillary endothelial cells were damaged, as indicated by gaps, in the THS rats. In comparison, pretreatment with PHC resulted in a marked attenuation of these pathological alterations.

Effects of PHC on TLR4 and p-p38MAPK protein expression in lung tissue, assessed by western blotting and immunofluorescence analysis. The present study assayed the effects of PHC on the activation of TLR4 and p-p38MAPK by western blotting. Following the THS challenge, TLR4 and p-p38MAPK expression increased, and PHC decreased the THS-induced expression of TLR4 and p-p38MAPK (Fig. 6A-C). p-p38MAPK expression in lung tissues was measured by 
A
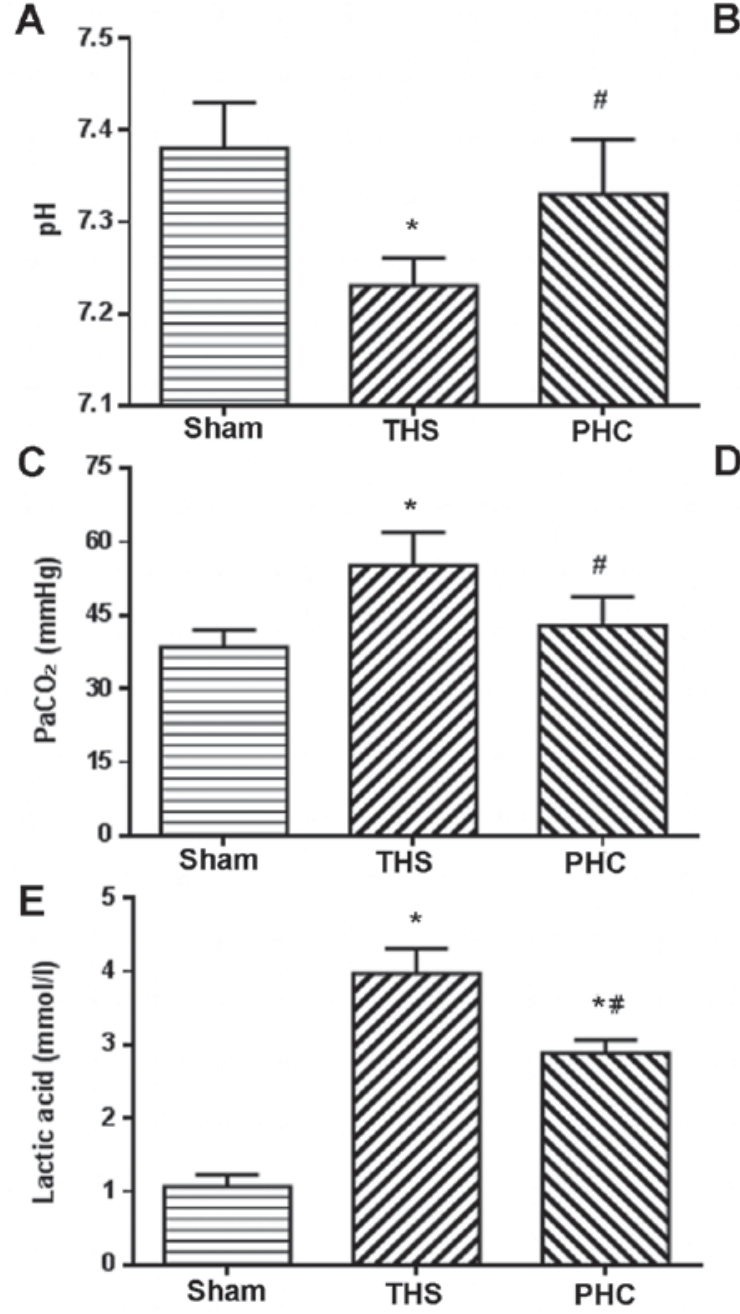

B
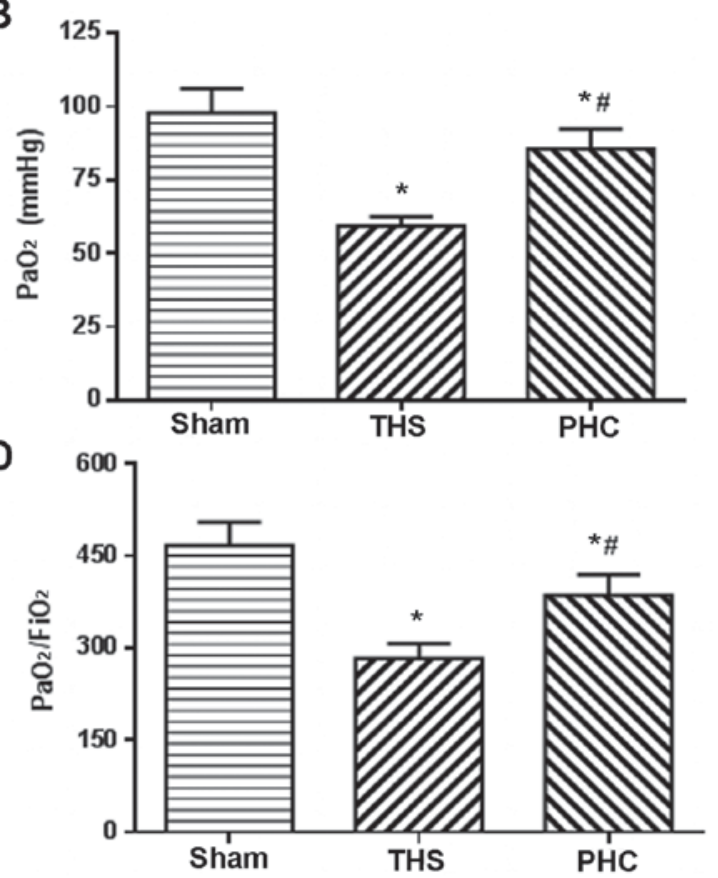

Figure 2. Alterations of blood gas levels and lactic acid in arterial blood gases. (A) $\mathrm{pH},(\mathrm{B}) \mathrm{PaO}_{2},(\mathrm{C}) \mathrm{PaCO}_{2},(\mathrm{D}) \mathrm{PaO}_{2} / \mathrm{FiO}_{2}$, and (E) lactic acid. ${ }^{*} \mathrm{P}<0.05$ vs. sham group; ${ }^{\#} \mathrm{P}<0.05$ vs. THS group. The data are presented as the mean \pm standard deviation $(\mathrm{n}=10)$. $\mathrm{Pa}$, partial pressure; FiO ${ }_{2}$, fraction of inspired oxygen; THS, blunt chest trauma with hemorrhagic shock; PHC, penehyclidine hydrochloride.
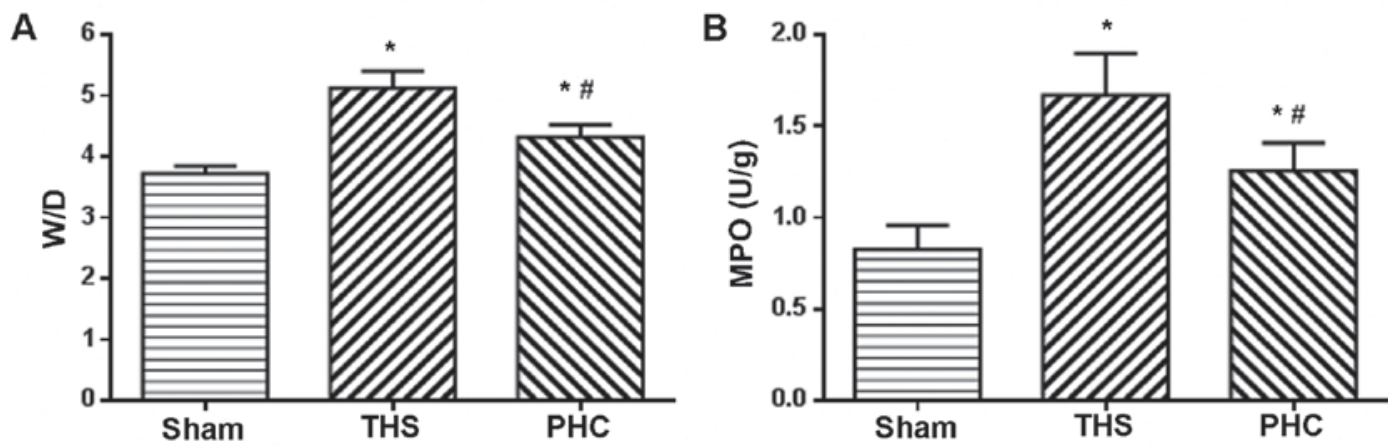

Figure 3. Lung W/D ratio and MPO activity. Compared with the results for the sham group, (A) the lung W/D weight ratio and (B) MPO activity were significantly increased at $6 \mathrm{~h}$ following ALI due to the THS challenge. ${ }^{*} \mathrm{P}<0.05$ vs. sham group; ${ }^{\mathrm{P}} \mathrm{P}<0.05 \mathrm{vs}$. THS group. The data are presented as the mean \pm standard deviation ( $\mathrm{n}=10)$. W/D, wet/dry weight; MPO, myeloperoxidase; THS, blunt chest trauma with hemorrhagic shock; PHC, penehyclidine hydrochloride; ALI, acute lung injury.

immunofluorescence analysis. In the sham group, positive cells were distributed in clumps with red colorization. In the THS rats, p-p38MAPK-positive cells were distributed throughout the visual fields, including alveolar macrophages, vascular endothelial cells, bronchial epithelial cells and interstitial cells, and were stained red. p-p38MAPK expression in tissues was increased following the THS challenge compared with that in the sham controls $(\mathrm{P}<0.05)$. Compared with the THS group, PHC administration inhibited p-p38MAPK expression in tissues (Fig. 6D and E). 
A Sham

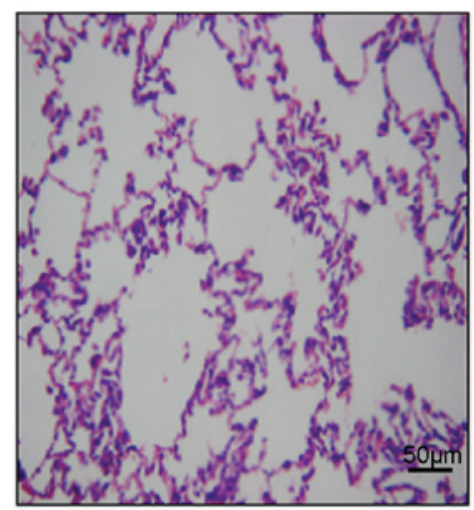

THS

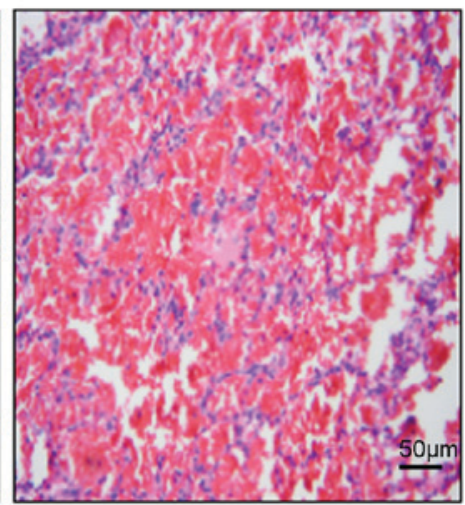

PHC

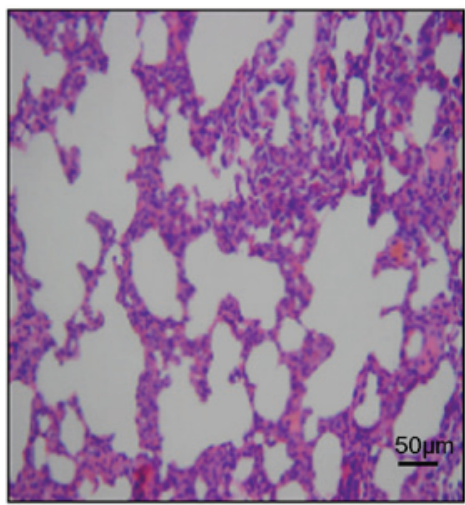

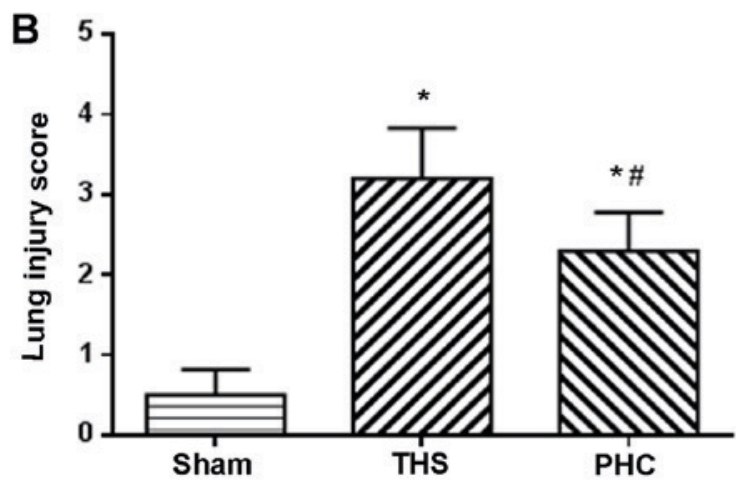

Figure 4. Pulmonary hematoxylin and eosin staining. (A) Microscopic findings of lung inflammation in lung tissues stained with hematoxylin and eosin (original magnification, $\mathrm{x} 200$ ). The THS group exhibited disruption to the normal alveolar structure, with severe congestion and hemorrhage associated with inflammatory cell infiltration. The PHC group exhibited comparatively mild inflammatory cellular infiltration and intra-alveolar hemorrhage. (B) Lung injury scores in each group. ${ }^{*} \mathrm{P}<0.05$ vs. sham group; ${ }^{*} \mathrm{P}<0.05$ vs. THS group. The data are presented as the mean \pm standard deviation ( $\mathrm{n}=10$ ). THS, blunt chest trauma with hemorrhagic shock; PHC, penehyclidine hydrochloride.
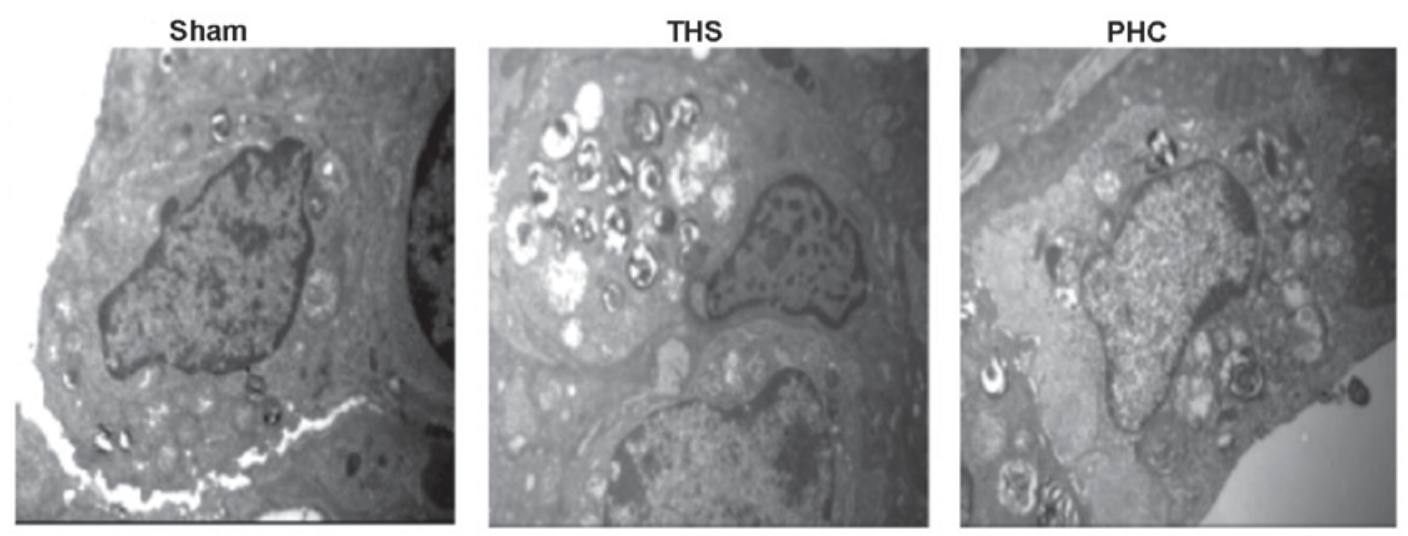

Figure 5. Detection of lung injury by transmission electron microscopy (original magnification, $\mathrm{x} 10,000$ ). In the sham group, the edge of the nuclear membrane was clear. The THS group exhibited significant pulmonary injury (degranulation of pulmonary alveolar type II cells, emptied osmiophilic lamellar bodies and obscured or disappeared cellular ridge). In the PHC group, a comparatively greater number of lamellar bodies was evident. THS, blunt chest trauma with hemorrhagic shock; PHC, penehyclidine hydrochloride.

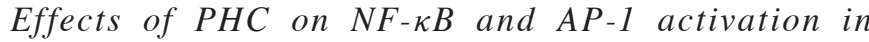
THS-induced ALI rats. NF- $\kappa \mathrm{B}$, a transcription factor known to regulate inflammatory gene expression in macrophages, is additionally considered to be a marker of classically activated pro-inflammatory macrophages. The role of PHC in the activation of $\mathrm{NF}-\kappa \mathrm{B}$ and AP-1 nuclear binding activity, due to THS-induced ALI, was analyzed. In response to THS, the DNA binding activity of NF- $\mathrm{NB}$ and AP-1 in lung tissues was significantly increased compared with those in sham group rats $(\mathrm{P}<0.05)$. PHC administration efficiently attenuated the increases in $\mathrm{NF}-\kappa \mathrm{B}$ and $\mathrm{AP}-1$ activity induced by THS ( $\mathrm{P}<0.05$; Fig. 7).

Effect of PHC on serum TNF- $\alpha, I L-6$ and $I L-1 \beta$ expression levels in THS-induced ALI rats. The serum expression levels of TNF- $\alpha$, IL- 6 and IL-1 $\beta$ were evaluated by ELISA, since 
A
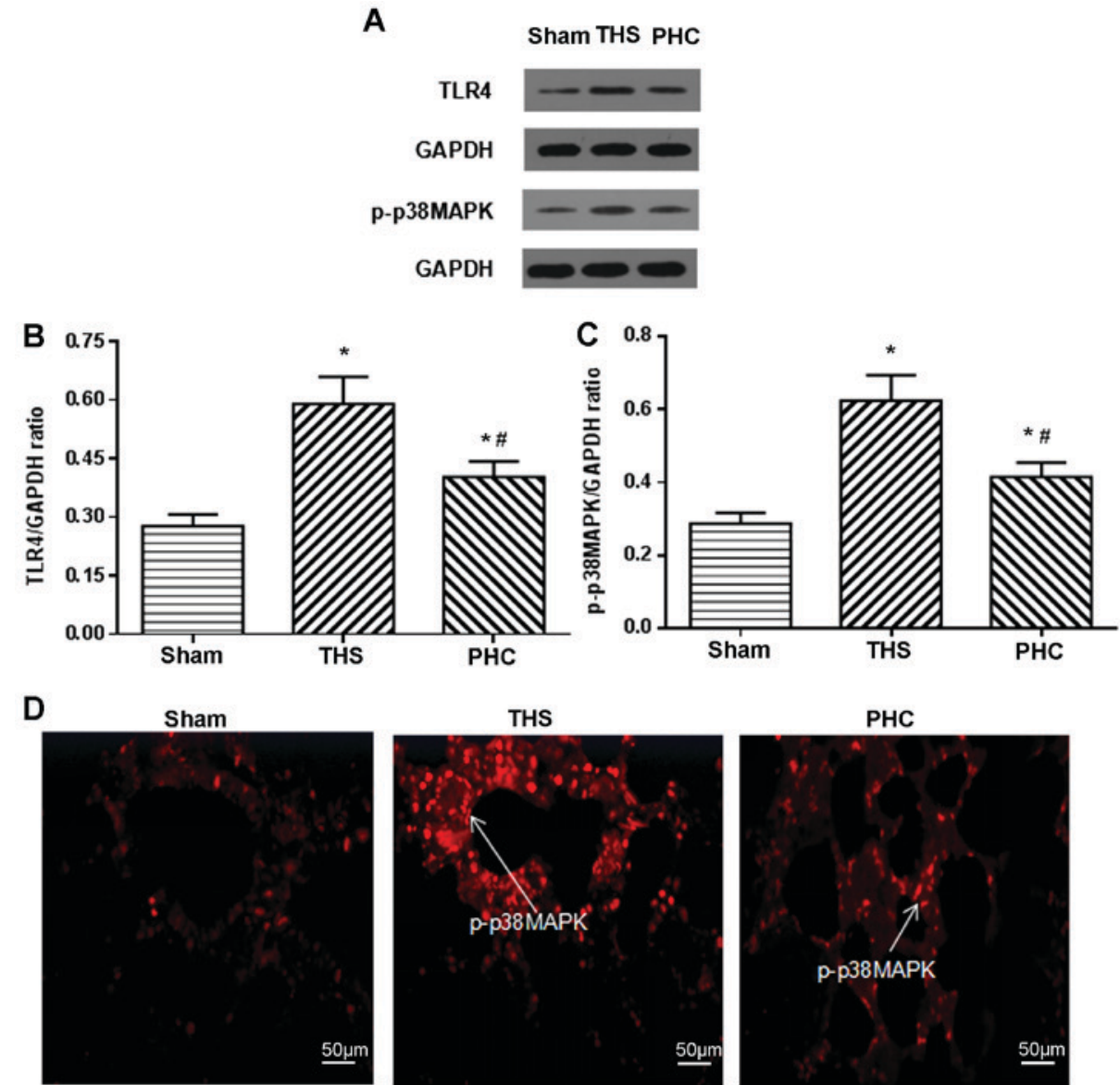

THS
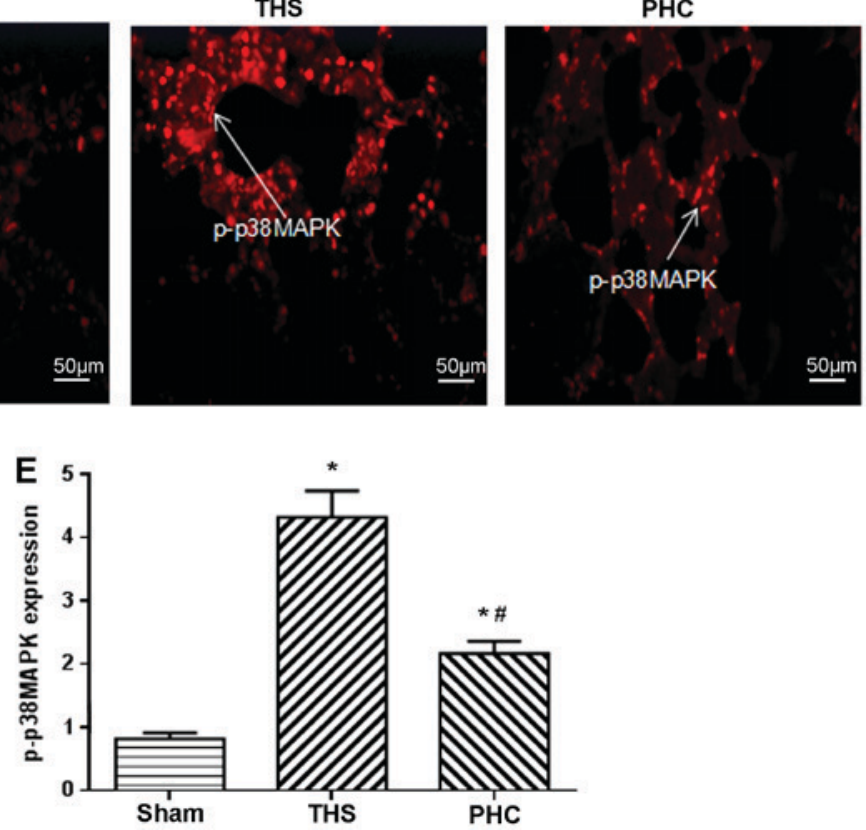

Figure 6. PHC treatment inhibits the THS-induced expression of TLR4 and p-p38MAPK. (A) Western blot analysis revealed that the expression of (B) TLR4 and (C) p-p38MAPK in the THS group was increased compared with that in the sham group. PHC attenuated the increase in the expression of TLR4 and p-p38MAPK compared with that in the THS group. The expression of p-p38MAPK in the lung tissue was examined under a light microscope following (D) the immunofluorescence assay (magnification, $\mathrm{x} 200$ ), and (E) the expression was quantified. " $\mathrm{P}<0.05$ vs. sham group; ${ }^{\mathrm{P}}<0.05$ vs. THS group. The data are presented as the mean \pm standard deviation $(n=10)$. THS, blunt chest trauma with hemorrhagic shock; PHC, penehyclidine hydrochloride; $\mathrm{p}$, phosphorylated; MAPK, mitogen-activated protein kinase; TLR4, Toll-like receptor 4.
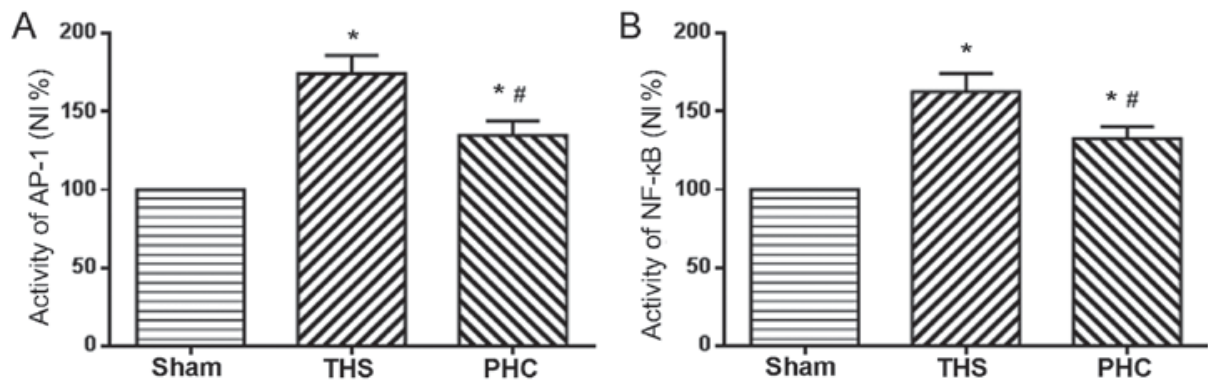

Figure 7. Effects of PHC treatment on alterations in NF- $\mathrm{kB}$ and AP-1 activation in the lungs of rats subjected to THS. Electrophoretic mobility shift assay analysis demonstrated decreased activity of (A) NF- $\mathrm{kB}$ and (B) AP-1 in the lung tissues of the sham group. By contrast, significantly higher activity levels of NF- $\mathrm{KB}$ and AP-1 were observed in the THS group. Compared with the THS group, the activity of NF- $\mathrm{kB}$ and AP-1 was decreased in lung tissues from the PHC group. "P $<0.05$ vs. sham group; ${ }^{\#} \mathrm{P}<0.05$ vs. THS group. The data are presented as the mean \pm standard deviation ( $\mathrm{n}=10$ ). THS, blunt chest trauma with

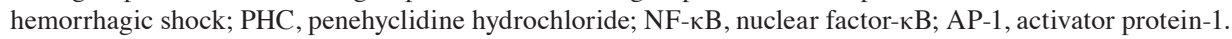



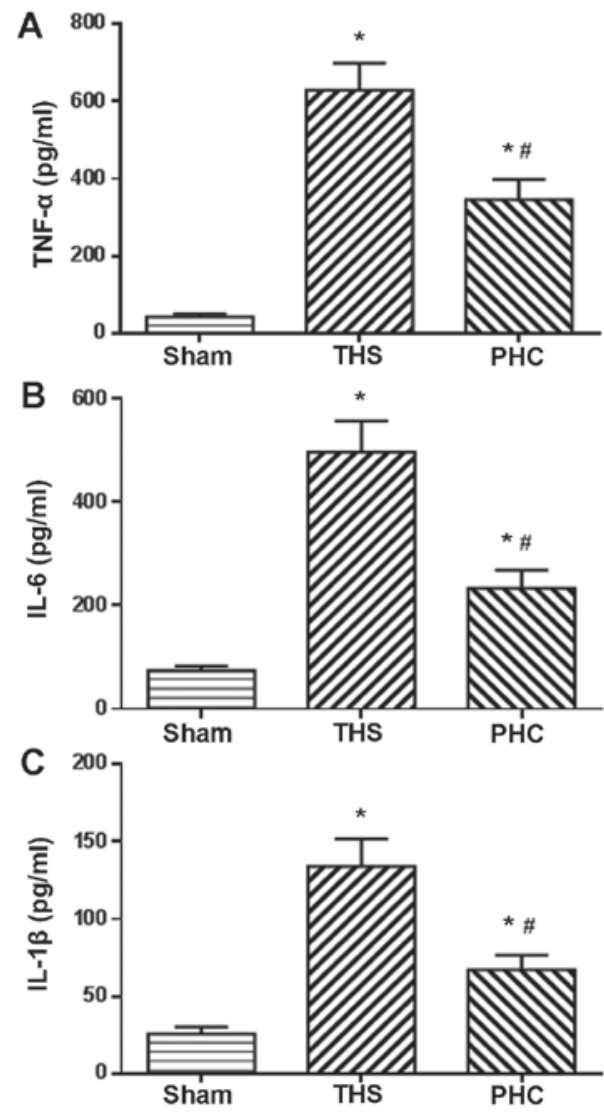

Figure 8. Effects of PHC treatment on alterations in the serum levels of TNF- $\alpha$, IL- 6 and IL- $1 \beta$ in THS-induced acute lung injury. Compared with the results for the sham group, serum TNF- $\alpha$, IL- 6 and IL- $1 \beta$ levels were significantly increased following THS challenge. Treatment with PHC significantly reduced TNF- $\alpha$, IL- 6 and IL- $1 \beta$ levels due to THS. The parameters depicted are (A) TNF- $\alpha$, (B) IL-6 and (C) IL- $1 \beta$. " $\mathrm{P}<0.05$ vs. sham group; ${ }^{*} \mathrm{P}<0.05$ vs. THS group. The data are presented as the mean \pm standard deviation $(n=10)$ THS, blunt chest trauma with hemorrhagic shock; PHC, penehyclidine hydrochloride; TNF- $\alpha$, tumor necrosis factor- $\alpha$; IL, interleukin.

the enhanced expression of these pro-inflammatory cytokines has been demonstrated to be associated with the activation of innate immunity. The serum TNF- $\alpha$, IL- 6 and IL- $1 \beta$ expression levels were significantly increased post-THS challenge, as presented in Fig. 8. Treatment with PHC significantly reduced the serum expression levels of TNF- $\alpha$, IL- 6 and IL- $1 \beta$ induced by THS $(\mathrm{P}<0.05$; Fig. 8$)$.

\section{Discussion}

ALI and ARDS following blunt chest trauma are important contributors to increased morbidity and mortality among patients worldwide (15). HS is a principal cause of mortality in the context of blunt chest trauma (16). ALI and ARDS are well-known common causes of pathogenesis following THS, and lead to an uncontrollable systemic inflammatory response $(17,18)$. The present study used a novel rat model of THS and investigated the effects of PHC on the TLR4 signaling pathway during ALI, including inflammation and lung damage. At $6 \mathrm{~h}$ post-THS challenge, the rats exhibited marked hypotension, arterial hypoxemia, leukocytosis and alveolar edema in the interstitial capillaries, and alveolar hemorrhage in histological assessments, which is consistent with the findings of Wu et al (12). Additionally, $\mathrm{PaO}_{2} / \mathrm{FiO}_{2}$ ratios $\leq 300 \mathrm{mmHg}$ were measured in THS rats, indicating that the replication of the two-hit-induced ALI model in the rats was successful.

Hoth et al (19) demonstrated that pulmonary inflammatory responses and systemic inflammatory responses are important characteristics post-THS-induced pulmonary contusion, including activated innate immune responses and an overwhelming release of pro-inflammatory cytokines, particularly TLR4, which serves a pivotal role in the host defense against invading pathogenic microorganisms and the recognition of pathogen-associated molecular patterns. A number of studies have demonstrated that TLR4 is an important pattern recognition receptor involved in the internal and external-derived inflammatory factor-mediated pulmonary inflammatory response $(20,21)$. TLR4 activation has been demonstrated to be involved in the pathogenesis of ALI and in the activation of numerous intracellular signaling systems, including the p38MAPK and NF- $\mathrm{kB}$ pathways, all of which are crucial regulators of inflammatory responses $(22,23)$. Barrenschee et al (24) reported that activation of p38MAPK by TLR4 upregulated inflammation-associated gene expression and stimulated the release of inflammatory cytokines. Yang et al (25) revealed that TLR4 mediated the activation of p38MAPK and contributed to high mobility group protein B1-induced ALI following liver ischemia/reperfusion injury. Furthermore, AP-1 is an important transcription factor required for the expression of numerous pro-inflammatory cytokines involved in the pathogenesis of ALI. Therefore, the present study aimed to determine potential therapeutic avenues for ALI with respect to the TLR4 signaling pathway. The results of the present study suggested that TLR4 may serve an important role in the pathophysiological mechanisms of THS-induced ALI and inflammation, and that p38MAPK, NF- $\mathrm{kB}$ and AP-1 may be involved in TLR4 signaling pathway-mediated lung inflammatory processes during THS.

PHC is a novel anticholinergic agent with antimuscarinic and antinicotinic activity, although it has little effect on HR and myocardial oxygen consumption. Animal and clinical studies have demonstrated that PHC selectively blocks muscarinic acetylcholine receptors 1 and $2\left(\mathrm{M}_{1}\right.$ and $\mathrm{M}_{2}$ ), and the nicontinic acetylcholine receptor, with fewer $\mathrm{M}_{2}$ receptor-associated cardiovascular side effects compared with other hyoscyamine medicines marketed in China $(26,27)$. Previous studies reported that PHC may have potential positive effects on lung/liver/renal injury, sepsis/septic shock, and ischemia/reperfusion injury (28-32). In the present study, it was demonstrated that pretreatment with PHC attenuated the development of lung damage, as indicated by a significantly elevated MAP, a stabilized HR, improved pulmonary oxygenation, and decreased MPO activity, W/D ratio and serum levels of TNF- $\alpha$, IL- 6 and IL- $1 \beta$, in addition to the activation of their transcription factors, NF- $\mathrm{KB}$ and AP-1, in the lung. These are important advances regarding the effects of PHC.

A number of studies have confirmed that PHC exerts anti-inflammatory, anti-apoptotic and antioxidative stress effects in animal injury models. In 2009, Shen et al (33) reported that PHC administration markedly attenuated the upregulation of the lung inflammatory response and decreased lung vascular leakage in a rat model of lipopolysaccharide-induced ALI. In 
2010, Wang et al (34) confirmed that PHC may alleviate lung injury by inhibiting the apoptosis regulator Bax/apoptosis regulator Bcl-2 signaling pathway in traumatic ALI. In 2011, Zhan et al (9) revealed that PHC was able to reduce inflammatory cytokine expression in septic mice. In 2012, Shu et al (35) demonstrated that PHC may decrease the expression levels of pro-inflammatory cytokines in the plasma following cardiopulmonary bypass. In 2013, Wang et al (36) discovered that PHC attenuated oxidative stress, the inflammatory response and apoptosis induced by renal ischemia/reperfusion in rats. The findings of the present study that the pro-inflammatory cytokine (TNF- $\alpha$, IL- 6 and IL-1 $\beta$ ) responses induced by THS were significantly attenuated by PHC, indicated that PHC has potent anti-inflammatory effects in ALI due to THS.

In conclusion, the results of the present study suggested that the activation of the TLR4 signaling pathway is a potentially important pathophysiological mechanism during the development of ALI following THS. These results indicate that PHC has anti-inflammatory properties and exerts a protective effect against the damage in ALI caused by THS, by inhibiting the TLR4 signaling pathway. Therefore, activation of the TLR4 signaling pathway may be one of the pathophysiological mechanisms of ALI, and PHC therapy may be a treatment option for inflammatory diseases, including ALI, in the future.

\section{Acknowledgements}

The authors would like to thank Shenzhen IVY-Valued Biotechnology Co. Ltd. (Shenzhen, China) for English editing service.

\section{Funding}

This research was supported by the National Natural Science Foundation of China (grant nos. 81671891 and 81401574) and the Natural Science Foundation of Hubei Province of China (grant no. 2016CFB251).

\section{Availability of data and materials}

All data generated or analyzed during this study are included in this published article.

\section{Authors' contributions}

XW, XS and ZX designed the study. XW, BZ, YL and EW performed the experiments. HL, LZ and QM analyzed the data. XW and HL wrote the manuscript.

\section{Ethics approval and consent to participate}

All animal experiments complied with the Guide for the Care and Use of Laboratory Animals of the National Institutes of Health, and were approved by the Bioethics Committee of Renmin Hospital of Wuhan University (Wuhan, China).

\section{Consent for publication}

Not applicable.

\section{Competing interests}

The authors declare that they have no competing interests.

\section{References}

1. Hildebrand F, Weuster M, Mommsen P, Mohr J, Fröhlich M, Witte I, Keibl C, Ruchholtz S, Seekamp A, Pape HC, et al: A combined trauma model of chest and abdominal trauma with hemorrhagic shock-description of a new porcine model. Shock 38: 664-670, 2012.

2. Erickson SE, Martin GS, Davis JL, Matthay MA and Eisner MD; NIH NHLBI ARDS Network: Recent trends in acute lung injury mortality: 1996-2005. Crit Care Med 37: 1574-1579, 2009.

3. Reino DC, Palange D, Feketeova E, Bonitz RP, Xu DZ, Lu Q, Sheth SU, Peña G, Ulloa L, De Maio A, et al: Activation of toll-like receptor 4 is necessary for trauma hemorrhagic shock-induced gut injury and polymorphonuclear neutrophil priming. Shock 38: 107-114, 2012.

4. Liu Z, Li Y, Liu B, Deperalta DK, Zhao T, Chong W, Duan X, Zhou P, Velmahos GC and Alam HB: Synergistic effects of hypertonic saline and valproic acid in a lethal rat two-hit model. J Trauma Acute Care Surg 74: 991-997, 2013.

5. Bosmann M, Russkamp NF and Ward PA: Fingerprinting of the TLR4-induced acute inflammatory response. Exp Mol Pathol 93: 319-323, 2012.

6. Wang L, Li Z, Zhang X, Wang S, Zhu C, Miao J, Chen L, Cui L and Qiao H: Protective effect of shikonin in experimental ischemic stroke: Attenuated TLR4, p-p38MAPK, NF- $\kappa$ B, TNF- $\alpha$ and MMP-9 expression, up-regulated claudin-5 expression, ameliorated BBB permeability. Neurochem Res 39: 97-106, 2014.

7. Joh EH, Gu W and Kim DH: Echinocystic acid ameliorates lung inflammation in mice and alveolar macrophages by inhibiting the binding of LPS to TLR4 in NF- $\kappa \mathrm{B}$ and MAPK pathways. Biochem Pharmacol 84: 331-340, 2012.

8. Han XY, Liu H, Liu CH, Wu B, Chen LF, Zhong BH and Liu KL: Synthesis of the optical isomers of a new anticholinergic drug, penehyclidine hydrochloride (8018). Bioorg Med Chem Lett 15: 1979-1982, 2005.

9. Zhan J, Liu Y, Zhang Z, Chen C, Chen K and Wang Y: Effect of penehyclidine hydrochloride on expressions of MAPK in mice with CLP-induced acute lung injury. Mol Biol Re 38: 1909-1914, 2011.

10. Li H, Qian Z, Li J, Han X and Liu M: Effects of early administration of a novel anticholinergic drug on acute respiratory distress syndrome induced by sepsis. Med Sci Monit 11: BR319-BR325, 2011.

11. Li BQ, Sun HC, Nie SN, Shao DB, Liu HM and Qian XM: Effect of penehyclidine hydrochloride on patients with acute lung injury and its mechanisms. Chin J Traumatol 13: 329-335, 2010.

12. Wu XJ, Xia ZY, Wang LL, Luo T, Zhan LY, Meng QT and Song XM: Effects of penehyclidine hydrochloride on pulmonary contusion from blunt chest trauma in rats. Injury 43: 232-236, 2012.

13. Raghavendran K, Davidson BA, Helinski JD, Marschke CJ, Manderscheid P, Woytash JA, Notter RH and Knight PR: A rat model for isolated bilateral lung contusion from blunt chest trauma. Anesth Analg 101: 1482-1489, 2005.

14. Knöferl MW, Angele MK, Diodato MD, Schwacha MG, Ayala A Cioffi WG, Bland KI and Chaudry IH: Female sex hormones regulate macrophage function after trauma-hemorrhage and prevent increased death rate from subsequent sepsis. Ann Surg 235: 105-112, 2002.

15. Liu Y, Du DY, Hu X, Xia DK, Xiang XY, Huang C, Zhou JH and Jiang JX: Prevalence and mortality of severe chest trauma in Three Gorges Area of China. Zhongguo Yi Xue Ke Xue Yuan Xue Bao 34: 567-572, 2012 (In Chinese).

16. Seitz DH, Perl M, Liener UC, Tauchmann B, Braumüller ST, Brückner UB, Gebhard F and Knöferl MW: Inflammatory Alterations in a novel nombination model of blunt chest trauma and hemorrhagic Shock. J Trauma 70: 189-196, 2011.

17. Bhatia M, Zemans RL and Jeyaseelan S: Role of chemokines in the pathogenesis of acute lung injury. Am J Respir Cell Mol Biol 46: 566-572, 2012.

18. Wohlauer M, Moore EE, Silliman CC, Fragoso M, Gamboni F, Harr J, Accurso F, Wright F, Haenel J, Fullerton D and Banerjee A: Nebulized hypertonic saline attenuates acute lung injury following trauma and hemorrhagic shock via inhibition of matrix metalloproteinase-13. Crit Care Med 40: 2647-2653, 2012. 
19. Hoth JJ, Wells JD, Brownlee NA, Hiltbold EM, Meredith JW, McCall CE and Yoza BK: Toll-like receptor 4-dependent responses to lung injury in a murine model of pulmonary contusion. Shock 31: 376-381, 2009.

20. Reino DC, Pisarenko V, Palange D, Doucet D, Bonitz RP, Lu Q, Colorado I, Sheth SU, Chandler B, Kannan KB, et al: Traumahemorrhagic shock-induced lung injury involves a gut-lymph-induced TLR4 pathway in mice. PLoS One 6: e14829, 2011.

21. Feinman R, Deitch EA, Aris V, Chu HB, Abungu B, Caputo FJ, Galante A, Xu D, Lu Q, Colorado I, et al: Molecular signatures of trauma-hemorrhagic shock-induced lung injury. Shock 28 360-368, 2007.

22. Chen C, Wang Y, Zhang Z, Wang $\mathrm{C}$ and Peng M: Toll-like receptor 4 regulates heme oxygenase-1 expression after hemorrhagic shock induced acute lung injury in mice: Requirement of p38 mitogen-activated protein kinase activation. Shock 31: 486-492, 2009

23. Yu HP, Hwang TL, Hsieh PW and Lau YT: Role of estrogen receptor-dependent upregulation of p38 MAPK/heme oxygenase 1 in resveratrol-mediated attenuation of intestinal injury after trauma-hemorrhage. Shock 35: 517-523, 2011.

24. Barrenschee M, Lex D and Uhlig S: Effects of the TLR2 agonists MALP-2 and Pam3Cys in isolated mouse lungs. PLoS One 5: e13889, 2010.

25. Yang Z, Deng Y, Su D, Tian J, Gao Y, He Z and Wang X: TLR4 as receptor for HMGB1-mediated acute lung injury after liver ischemia/reperfusion injury. Lab Invest 93: 792-800, 2013.

26. Wang YA, Zhou WX, Li JX, Liu YQ, Yue YJ, Zheng JQ, Liu KL and Ruan JX: Anticonvulsant effects of phencynonate hydrochloride and other anticholinergic drugs in soman poisoning: Neurochemical mechanisms. Life Sci 78: 210-223, 2005.

27. Xiao HT, Liao Z, Meng X, Yan X, Chen S and Mo Z: Effects of the selective muscarinic receptor antagonist penehyclidine hydrochloride on the respiratory tract. Pharmazie 64: 337-341, 2009.

28. Li J, Li J, Zhang L, Huang Y, Pan JH and Chen KZ: Penehyclidine prevents nuclear factor-KappaB activation in acute lung injury induced by lipopolysaccharide. J Pharm Pharmaco 60: 1197-1205, 2008.
29. Cai DS, Jin BB, Pei L and Jin Z: Protective effects of penehyclidine hydrochloride on liver injury in a rat cardiopulmonary bypass model. Eur J Anaesthesiol 27: 824-828, 2010.

30. He SS, Lin CS, Gu MN, Chen DT, Zong SL and Chen Y: Protective effects of penehyclidine hydrochloride against acute renal injury induced by hemorrhagic shock and lipopolysaccharides in rats. Nan Fang Yi Ke Da Xue Xue Bao 31: 899-902, 2011 (In Chinese).

31. Zhan J, Wang YL, Wang CY, Li JG, Zhang ZZ and Jia BH: Protective effects of penehyclidine hydrochloride on septic mice and its mechanism. Shock 28: 727-732, 2007.

32. Zhang Y, Leng YF, Xue X, Zhang Y, Wang T and Kang YQ: Effects of penehyclidine hydrochloride in small intestinal damage caused by limb ischemia-reperfusion. World J Gastroentero 17: 254-259, 2011.

33. Shen W, Gan J, Xu S, Jiang G and Wu H: Penehyclidine hydrochloride attenuates LPS-induced acute lung injury involvement of NF-kappaB pathway. Pharmacol Res 60: 296-302, 2009.

34. Wang LL, Zhan LY, Wu XJ and Xia ZY: Effects of penehyclidine hydrochloride on apoptosis of lung tissues in rats with traumatic acute lung injury. Chinese J Traumatol 13: 15-19, 2010.

35. Shu LJ and Wei XC: Effect of Penehyclidine hydrochloride in systemic inflammatory response syndrome caused by cardiopulmonary bypass. Sichuan Da Xue Xue Bao Yi Xue Ban 43: 543-546, 2012.

36. Wang YP, Li G, Ma LL, Zheng Y, Zhang SD, Zhang HX, Qiu M and Ma X: Penehyclidine hydrochloride ameliorates renal ischemia-reperfusion injury in rats. J Surg Res 186: 390-397, 2014. 